\title{
Ablative Laser Propulsion Using Multi-Layered Material Systems
}

\author{
M. Nehls, D. Edwards, P. Gray, and T. Schneider \\ Materials, Processes, and Manufacturing Department, \\ NASA/Marshall Space Flight Center, MSFC, AL 35812
}

Experimental investigations are ongoing to study the force imparted to materials when subjected to laser ablation. When a laser pulse of sufficient energy density impacts a material, a small amount of the material is ablated. A torsion balance is used to measure the momentum produced by the ablation process. The balance consists of a thin metal wire with a rotating pendulum suspended in the middle. The wire is fixed at both ends as shown in figure 1 .

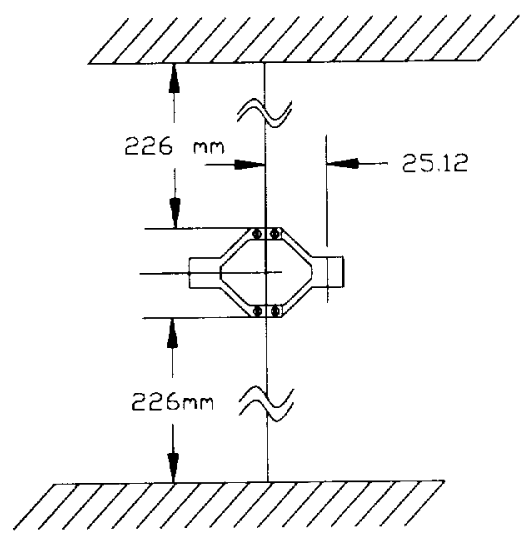

Figure 1. Diagram of the Torsion Balance Used to Measure Momentum Produced by Material Ablation.

The wire of a known torsion spring constant allows calculation of the maximum impulse produced by the ablation. The displacement of the pendulum is measured to calculate the momentum, which is the product of the average force and the time interval from the zero position to the maximum displacement position. The impulse varies as a function of the angular displacement of the pendulum, with the impulse defined as zero at zero displacement and a maximum at the maximum angular displacement, $\theta_{\max }$. Figure 2 diagrams the angular displacement parameters for the torsion balance. 


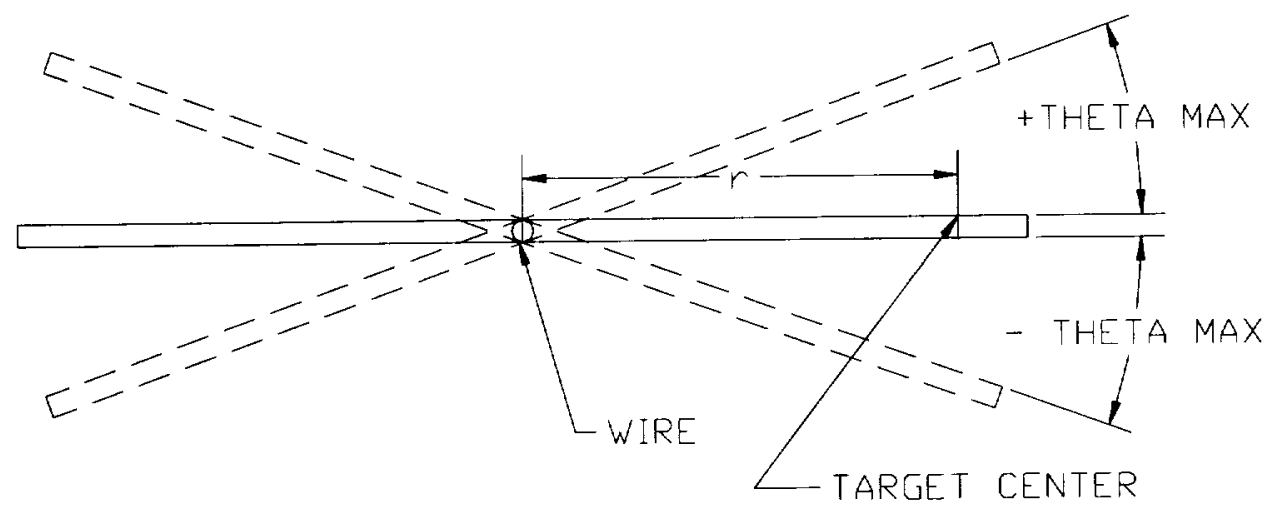

Figure 2. Diagram of the Torsion Balance Defining the Radius and Angular Displacement Parameters Used for Momentum Calculations.

The average force is defined by:

$$
\mathrm{F}_{\mathrm{ave}}=\mathrm{C} \theta_{\max } / 2 \mathrm{r}
$$

where $\mathrm{C}$ is the torsion spring constant, $\mathrm{r}$ is the distance from the center of the laser impact sight to the center of the pendulum, and $\theta_{\max }$ is the angle of displacement in radians. The momentum change is calculated by using the relationship:

$$
\Delta \mathrm{p}=\mathrm{F}_{\mathrm{ave}} \Delta \mathrm{t}
$$

where $\Delta t$ is the time interval between the pendulum at rest and the maximum displacement position.

A common unit of measure to determine the efficiency of converting light energy to kinetic energy through the ablation process is the coupling coefficient. The coupling coefficient is the ratio of the transferred momentum to the laser power density. ${ }^{1}$ The laser power density is defined by:

$$
P=E / t A
$$

where $E$ is the laser energy, $t$ is the pulse width and $A$ is the cross sectional area of the beam. 
Coupling coefficients for several monolithic materials have been generated and presented in a previous publication ${ }^{2}$. Figure 3 plots the coupling coefficients versus the power densities for these monolithic materials.

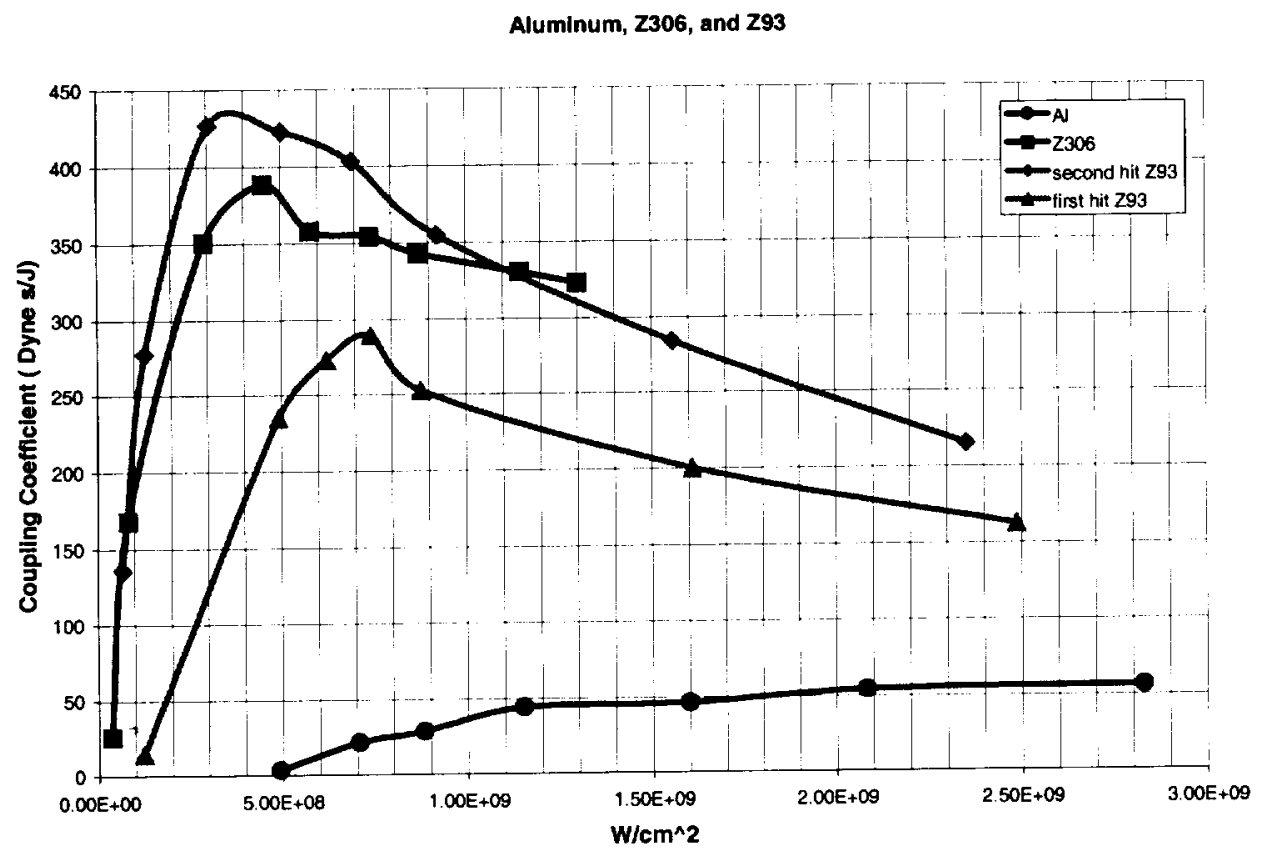

Figure 3. Coupling Coefficients for Aluminum, Z306 and Z93.

Recently, multi-layered material systems were investigated. These multi-layered materials were composed of a transparent front surface and opaque sub surface. The laser pulse penetrates the transparent outer surface with minimum photon loss and vaporizes the underlying opaque layer. It is possible that a plasma state exists during this interaction. Physically confining this vaporized gas between the opaque layer and the transparent surface leads to localized high pressure which eventually tears the transparent outer surface material. The vaporized gas is directionally vented producing impulse levels approximately an order of magnitude higher than those produced by monolithic surfaces. Results of impulse measurements from several multi-layered material systems will be presented. 


\section{References}

1. Campbell, J.W., "Project PRION: Orbital Debris Removal Using Ground-Based Sensors and Lasers", NASA Technical Memorandum - 108522, October 1996.

2. Gray, P.A. et.al., "Laser Ablative Force Measurements on Manmade Space Debris", AIAA-2001-0645, AIAA Conference, Reno Nevada, January 8-11, 2001. 